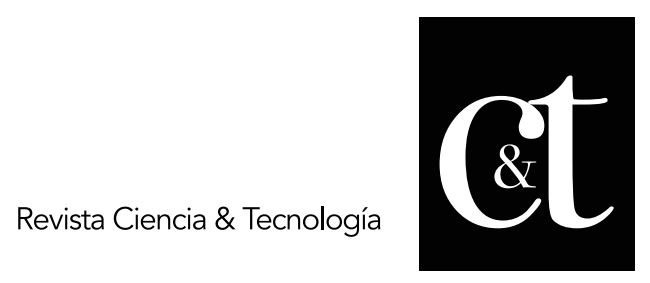

No. 32, 31 de octubre de 2021

ISSN impreso: 1390 - 6321

ISSN online: 2661 - 6734

\title{
Modelo de cloud computing en el control de inventarios para PYMEs del sector ferretero en la ciudad de Machala
}

\section{Cloud computing model in inventory control for SMEs in the hardware sector of the Machala city}

\author{
César Llaguno Vera ${ }^{1}$ \\ cesarllaguno@gmail.com \\ https://orcid.org/0000-0002-9054-4110 \\ Edwin León León ${ }^{2}$ \\ edwin.enrique88@gmail.com \\ https://orcid.org/0000-0002-5600-2203
}

Recibido: 28/6/2021, Aceptado: 28/9/2021

\begin{abstract}
RESUMEN
Se analiza la implementación del Cloud Computing como servicio en la nube para el control de inventarios en las pequeñas y medianas empresas (PYMEs) del sector ferretero de la ciudad de Machala, con el objetivo de establecer una guía de un modelo de adopción tecnológico TOE que ayude a mejorar la calidad de los procesos, reduzcan costos e incrementen ganancias. El diseño de la investigación es no experimental con alcance correlacional y enfoque cuantitativo. Se aplica una encuesta con escala Likert a una muestra aleatoria de 173 gerentes y propietarios de empresas del sector. Los resultados evidencian que únicamente el $4.05 \%$ de las empresas ha implementado Cloud Computing, con una predisposición de implementación del $69.36 \%$; el $71.7 \%$ considera que el cambio generará beneficios económicos, mientras que el $77 \%$ estima que mejorarán las habilidades internas y externas de la empresa. Las variables independientes analizadas dentro del contexto tecnológico y organizacional seleccionadas en el modelo TOE, demostraron ser altamente significativas con niveles entre 0.001-0.010, y valores positivos de Chi2 indicando la relación de estas variables con la variable dependiente "Adopción de Cloud Computing", comprobando que existe una percepción favorable del sector para su implementación en el control de inventarios.
\end{abstract}

Palabras clave: Información en la nube, PYMEs, TOE, sector ferretero, control de inventarios

\section{ABSTRACT}

${ }^{1}$ Magíster, Universidad Tecnológica Empresarial de Guayaquil, Ecuador.

${ }^{2}$ Magíster, Universidad Tecnológica Empresarial de Guayaquil, Ecuador. 


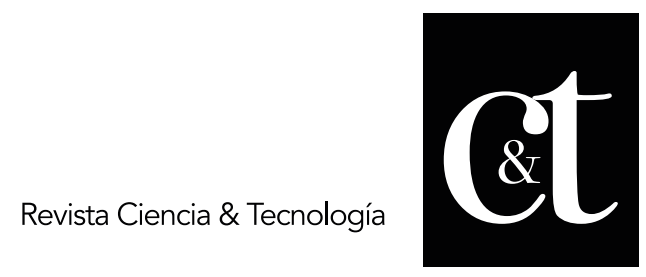

No. 32, 31 de octubre de 2021

ISSN impreso: 1390 - 6321

ISSN online: 2661 - 6734

The implementation of Cloud Computing as a cloud service for inventory control in small and medium-sized companies (SMEs) of the hardware sector of the city of Machala is analyzed, with the aim of establishing a guide for a TOE technology adoption model that help improve the quality of processes, reduce costs and increase profits. The research design is non-experimental with a correlational scope and a quantitative approach. A Likert scale survey is applied to a random sample of 173 managers and owners of companies in the sector. The results show that only $4.05 \%$ of the companies have implemented Cloud Computing, with an implementation predisposition of $69.36 \% ; 71.7 \%$ consider that the change will generate economic benefits, while $77 \%$ estimate that the internal and external skills of the compny will improve. The independent variables analyzed within the technological and organizational context selected in the TOE model, proved to be highly significant with levels between 0.001-0.010, and positive values of Chi2 indicating the relationship of these variables with the dependent variable "Adoption of Cloud Computing", checking that there is a favorable perception of the sector for its implementation in inventory control.

Keywords: Cloud Computing, SMEs, TOE, hardware store, inventory control

\section{Introduction}

Ercolani (2017) menciona que las empresas en periodo de crisis se ven en la necesidad de disminuir sus gastos y aumentar la eficacia en la implementación de sistemas informáticos, por ende, insertar un programa basado en la nube que facilite y agilite las operaciones, además de funcionar como un modelo económico para adquirir y administrar los recursos de las Tecnologías de la Información (TI), es realmente viable.

La Computación en la Nube o Cloud Computing, es la tecnología de "estos tiempos", ha permitido que las empresas funcionen en tiempo compartido para el desarrollo de sus actividades. Ercolani (2012).

El término Cloud Computing proviene de la idea de que las aplicaciones se venderían como servicios, insertada por John McCarthy en el año 1961, pero no es hasta el año 2006 que Google populariza el Cloud Computing creando un programa en la web gratuito para crear documentos en línea permitiendo la posibilidad de colaborar en grupo (Jimenez, 2013). Por otra parte, el Instituto Nacional de Estándares y Tecnología (NIST) define la computación en la nube como un modelo conveniente de acceso a la red a pedido o bajo demanda, donde sus recursos (redes, servidores, almacenamiento, aplicaciones y servicios), se encuentran como un conjunto de servicios a través de internet, que pueden ser configurables y manejables con poco esfuerzo de la administración o interacción del proveedor de servicio. NIST indica que este modelo de nube se compone de cinco características esenciales, tres modelos de servicio y cuatro modelos de implementación, como se observa en el gráfico 1 y describe a continuación (Mell y Grance, 2011). 


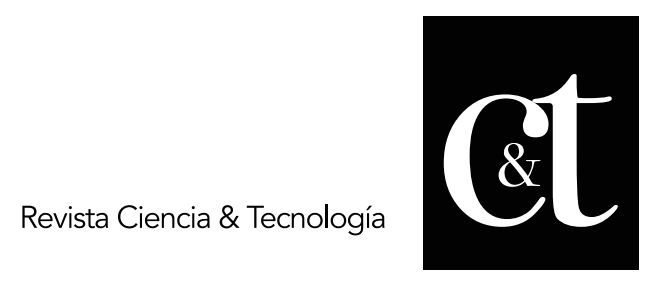

No. 32, 31 de octubre de 2021

ISSN impreso: 1390 - 6321

ISSN online: 2661 - 6734

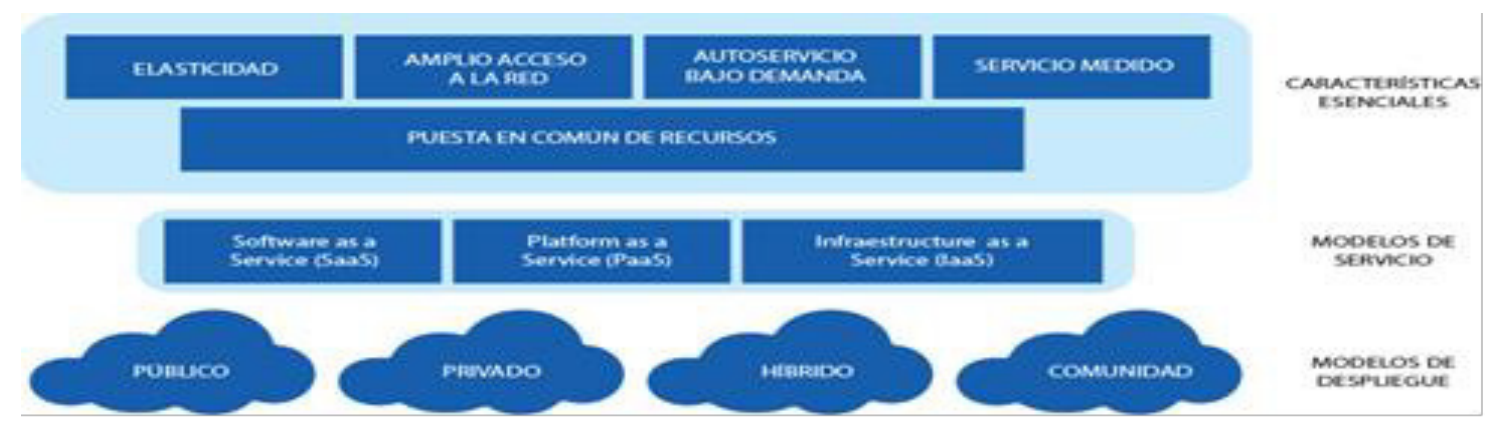

\section{Gráfico 1: Definición Cloud Computing}

Fuente: Elaboración propia

De los tres modelos de servicio que ofrece Cloud, Software como Servicio (SaaS) es el más idóneo para el caso de las PYMEs.

Aljabre (2012), indica que la tecnología en la nube no necesita ser creada por la empresa que la usa; más bien lo proporciona una infraestructura de empresa en la nube. Ercolani (2017), estudia la adopción de Cloud Computing como un "programa" que se desprende de un modelo de despliegue SaaS, e indica que este tipo de modelos "utilizan la nube pública para su despliegue sin necesidad de hardware añadido o servidores en el interior de la empresa", lo cual es un gran beneficio para las PYMEs p. 4.

El Cloud Computing posee además otros grandes beneficios para las PYMEs, entre ellas: facilidad para innovar (Mariscal y Gil, 2013); escalabilidad en cuanto al aprovisionamiento de recursos (Vidhyalakshmi y Kumar, 2016); y análisis en tiempo real del comportamiento de los clientes (Dean y Saleh, 2010). Por otra parte, también se encuentran barreras que dificultan la adopción tal como: la falta de un socio-tecnológico adecuado que ofrezca acompañamiento y formación durante el proceso de adopción (Pérez, Solana y Trigueros, 2018); problemas de seguridad y miedo a la pérdida de datos confidenciales (Armbrust et al., 2010); y el costo de ancho de banda, calidad y velocidad de la conectividad (Mohlameane y Ruxwana, 2014).

La provincia de El Oro está conformada en su mayor parte por micro, pequeñas y medianas empresas; según estudio de Celleri, Rivas y Andrade (2018), sólo el $14 \%$ tienen características de grandes empresas y señalan que el $55.1 \%$ de las PYMEs desconocen la tecnología Cloud Computing, al $42.2 \%$ no les parece necesario migrar a los servicios de esta tecnología y el $22.4 \%$ tienen preocupaciones por el nivel del servicio, disponibilidad, integridad y responsabilidad de los proveedores.

Otro estudio revela que en la ciudad de Machala el $58 \%$ de las PYMEs utilizan software tradicionales para control de inventarios y facturación y el $79.3 \%$ disponen de un centro de cómputo propio básico (Mazon et al., 2018). En el análisis realizado por Alomoto, Acuña y Salvador (2014), enfocado en la eficiencia de las PYMEs del país se evidenció una menor relevancia en cuanto al manejo de inventarios. 
Con Cloud, las empresas tienen la alternativa de implementar sistemas registrándose y pagando por su uso, reduciendo gastos en mantenimiento de software e inclusive generando mayor flexibilidad para cambio de proveedor (Dubey y Wagle, 2007). Reducir costos es la motivación principal que tienen las empresas para cambiarse de un sistema ERP tradicional a un entorno en la nube (Ruivo, Rodrigues y Oliveira, 2015). Así como también la reducción de gastos en licencia de software, hardware, personal de TI, costos de mantenimiento y una mejor comunicación con socios externos (Salleh, Teoh y Chan, 2012).

Existen varias teorías y marcos para la adopción o uso de nuevas tecnologías; como: Teoría de la Acción Razonada (TRA), Teoría del Comportamiento Planificado (TPB), Modelo de Aceptación de Tecnología (TAM), marco de trabajo Tecnología, Organización y Entorno (TOE) y la Teoría de la Difusión de la Innovación (DOI), (Indahningrum, 2020), como se observa en el gráfico 2.

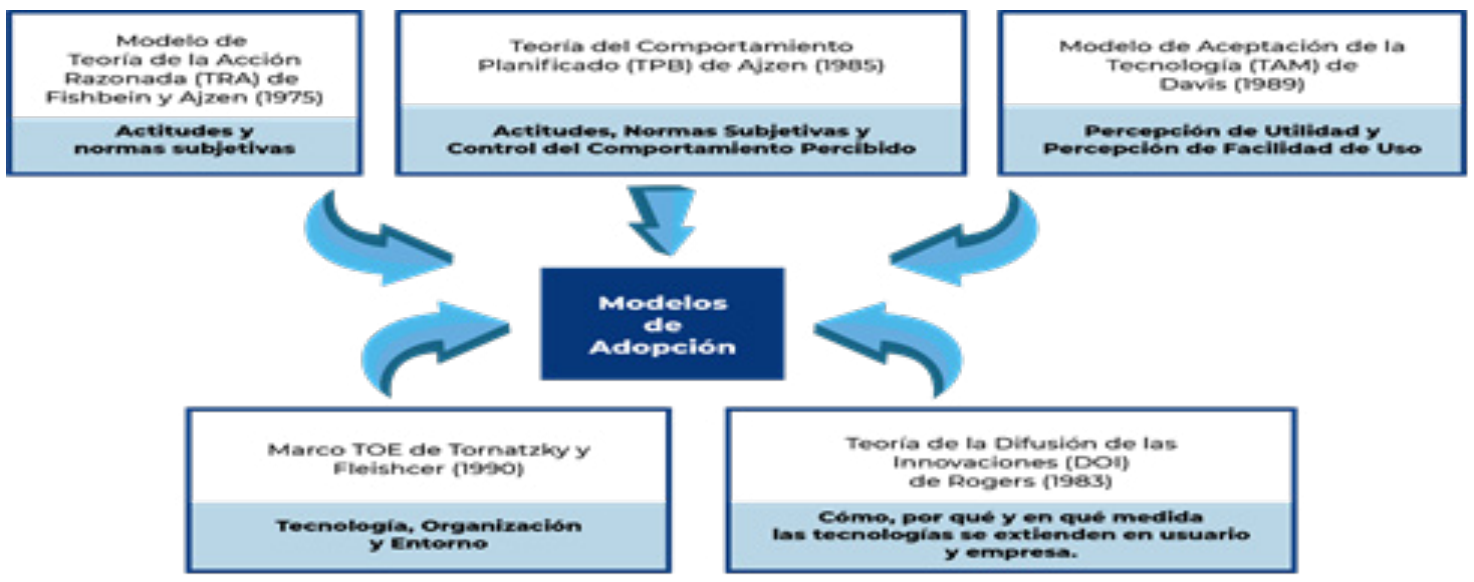

Gráfico 2: Modelos de adopción de tecnología

Fuente: Elaboración propia

El contexto tecnológico considera las tecnologías de la empresa como las existentes en el mercado; el contexto organizacional se refiere al alcance, tamaño y estructura de la empresa; y finalmente contexto del entorno, industria, competidores y relaciones con el gobierno; son los tres contextos que abarca el modelo TOE (gráfico 3) (Otake, 2019).

A nivel del sector y para la ejecución del presente trabajo se consideraron nueve variables independientes (gráfico 3 ). 


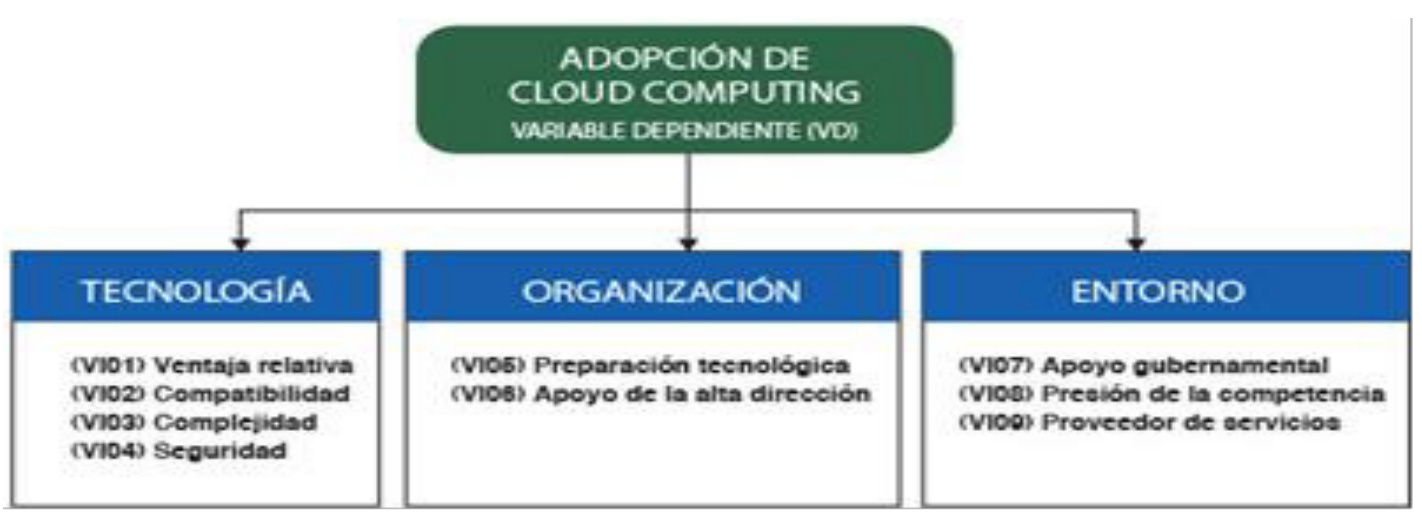

Gráfico 3: Variables aplicadas en el modelo TOE

Fuente: Elaboración propia

La elección de las variables se realizó a partir de la realidad actual de las PYMEs del sector ferretero; en el contexto tecnológico se agregó la variable "seguridad", la cual comúnmente no es considerada en los estudios, la razón de ello es la complicación que implica esta variable en el estudio o el poco interés que se le brinda; en el contexto organizacional las dos variables que se estudian corresponden a la preparación, conocimientos, recursos y apoyo de los altos mandos para la adopción de la tecnología; y relacionado al contexto del entorno, apoyo del gobierno, la competencia y proveedores son las variables más relevantes.

\section{Metodología}

El tipo de diseño de la investigación fue no experimental, puesto que se observó la conducta de las variables sin incidir en ellas para la obtención de los resultados, además fue de tipo descriptivo porque se investigó al grupo de estudio, variables y los distintos modelos para el uso de tecnologías. El alcance del trabajo fue correlacional con la finalidad de conocer el grado de relación de cada una de las variables, empleando el método deductivo que parte de las concepciones teóricas y se valida con la información producto del levantamiento de datos obtenidos por medio de la encuesta realizada a los gerentes o propietarios de las PYMEs.

La encuesta se realizó a 173 PYMEs del sector ferretero de la ciudad de Machala, datos que fueron obtenidos de la página web del Servicio de Rentas Internas (SRI), catastro del Registro Único de Contribuyentes (RUC), donde se encontraron registradas 312 empresas con la actividad "Venta al por menor de artículos de ferretería", de la cual mediante el cálculo de muestreo de población finita se determinó aplicar el instrumento solo para ese grupo de empresas, considerado como la muestra del presente trabajo.

El enfoque de la investigación es de tipo cuantitativo porque se recopilaron datos a través de una fuente primaria e instrumento utilizado en el trabajo como fueron las encuestas, a través de una escala de Likert aplicada que permitió la evaluación de las variables y fue ponderada mediante la escala de Fuzzy para el tratamiento de los resultados en el software estadístico; además, se utilizan fuentes secundarias tales como artículos científicos, tesis, libros y páginas web. 


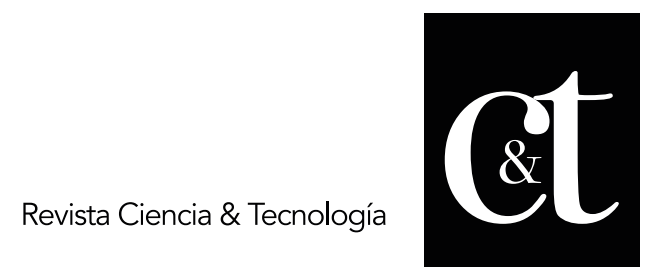

No. 32, 31 de octubre de 2021

ISSN impreso: 1390 - 6321

ISSN online: 2661 - 6734

Finalmente con el Software Estadístico SPSS V23, se realizó el análisis estadístico descriptivo de la situación actual de las PYMEs del sector en cuanto a la situación actual de las PYMEs en la adopción de Cloud Computing; conocimiento y problemas que poseen con sus sistemas de inventarios actuales; también se realizaron los respectivos análisis correlacionales individuales de cada variable independiente respecto a la variable dependiente "Adopción de Cloud Computing" a través del coeficiente Chi-Cuadrado y a su vez el análisis correlacional de las dimensiones consideradas en el estudio de cada variable independiente mediante el cálculo del coeficiente de Pearson. El análisis consideró un coeficiente de confianza del 95\% $y$ un coeficiente de error o nivel de significancia del 5\% ( $p$ - valor $\leq 0.05$ ).

\section{Resultados y discusión}

En la ciudad de Machala las PYMEs del sector ferretero están conformadas por $48.55 \%$ microempresas y $51.45 \%$ pequeñas empresas, de las cuales el $35.26 \%$ en el año 2020 indicaron haber obtenido ingresos estimados anuales menores de $\$ 100.000,00$, mientras que, el $30.64 \%$ y $34.10 \%$ entre $\$ 100.000,00$ a $\$$ $1^{\prime} 000.000,00$ y $\$ 1^{\prime} 000.000,00$ a $\$ 5^{\prime} 000.000,00$ respectivamente.

En cuanto a los sistemas de inventarios empleados en estas empresas el $72.26 \%$ utiliza plataformas tradicionales para el manejo de sus inventarios, el $20.81 \%$ utiliza hojas de cálculo Excel, el $5.20 \%$ lo realizan de manera manual y sólo el $4.05 \%$ ha implementado Cloud Computing.

De los encuestados, el $79.77 \%$ indicaron haberse enfrentado a problemas en sus sistemas actuales de control del stock de la mercadería y un pequeño grupo que corresponde al $20.23 \%$ parecen estar conformes.

Con la aplicación de la encuesta se logró observar la dificultad que tienen las PYMEs del sector para controlar de forma adecuada y eficiente su inventario con los sistemas que emplean actualmente, estos sistemas son rudimentarios y las empujan a caer en errores de cálculo y control, lo cual se ve reflejado en pérdidas económicas futuras.

Definitivamente estas acciones tienen una forma de justificarse y es "La falta de conocimiento", las empresas que ya han implementado Cloud Computing son minoría; del total de las PYMEs encuestadas solo el $23.12 \%$ conoce de esta tecnología, mientras que el $45.09 \%$ tiene algunos conocimientos de la nube y el $31.79 \%$ no posee conocimiento alguno.

Aunque el panorama no resulte ser tan optimista en cuanto a la adopción actual del Cloud Computing, se logró determinar que el $69.36 \%$ de los encuestados indicaron estar dispuestos a su adopción para el control de inventarios. Lo que demuestra, la gran aceptación que existe por parte de los propietarios, gerentes o encargados en implementar mejoras en sus sistemas de inventario mediante el uso de la tecnología; debido que las empresas se vieron en la necesidad de transformarse y asignar recursos a la implementación de herramientas actuales que permitan mejorar y trascender de lo tradicional a lo actual, con la finalidad de volverse más competitivas, sostenibles y rentables. 
Las variables independientes seleccionadas en el modelo demostraron ser altamente significativas con un nivel de significancia entre 0.001 y 0.010 ; los valores de Chi2 son positivos, indicando la relación de las variables independientes con la variable dependiente; Una excepción se refleja en la VI ventaja relativa, que arrojó un p-valor de 0.047, aproximado al 0.05; lo que indica, la variable a pesar que se encuentra relacionada con el modelo tiene una ponderación baja, se aproxima al error permitido pero está dentro de los parámetros que se establecieron en el estudio (tabla 1 ).

Tabla 1: Resultados del análisis correlacional de las variables independientes

\begin{tabular}{|c|c|c|c|c|c|c|c|c|c|}
\hline & \multicolumn{4}{|c|}{ Contexto Tecnológico } & & & & & \\
\hline & \multirow{2}{*}{ Variable Ind. } & \multicolumn{2}{|c|}{ Resultados } & \multirow{2}{*}{ Pond. } & & & & & \\
\hline & & p-valor & Chi2 & & & & & & \\
\hline \multirow{15}{*}{ 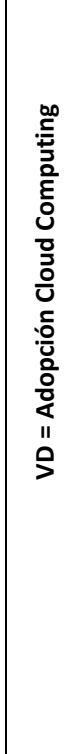 } & Ventaja Relativa & 0,047 & 9,636 & Baja & & & & & \\
\hline & Compatibilidad & 0,004 & 15,517 & Alta & & & & & \\
\hline & Complejidad & 0,001 & 19,969 & Alta & & & & & \\
\hline & Seguridad & 0,001 & 25,56 & Alta & & & & & \\
\hline & \multicolumn{4}{|c|}{ Contexto Organizacional } & & & & & \\
\hline & \multirow{2}{*}{ Variable Ind. } & \multicolumn{2}{|c|}{ Resultados } & \multirow{2}{*}{ Pond. } & & & & & \\
\hline & & p-valor & Chi2 & & & & & & \\
\hline & Preparación Tecnológica & 0,003 & 16,324 & Alta & & & & & \\
\hline & Apoyo de la Alta Dirección & 0,003 & 16,324 & Alta & \multicolumn{5}{|c|}{ Escala de medición de variables } \\
\hline & \multicolumn{4}{|c|}{ Contexto Tecnológico } & Escala & Criterio & Rango & & Valoración \\
\hline & \multirow{2}{*}{ Variable Ind. } & \multicolumn{2}{|c|}{ Resultados } & \multirow{2}{*}{ Pond. } & 1 & $5 \%$ & 0,0401 & 0,0500 & Baja \\
\hline & & p-valor & Chi2 & & 2 & $4 \%$ & 0,0301 & 0,0400 & Baja \\
\hline & Apoyo Gubernamental & 0,001 & 17,633 & Alta & 3 & $3 \%$ & 0,0201 & 0,0300 & Media \\
\hline & Presión de la Competencia & 0,001 & 13,308 & Alta & 4 & $2 \%$ & 0,0101 & 0,0200 & Media-Alta \\
\hline & Proveedor de Servicios & 0,004 & 13,564 & Alta & 5 & $1 \%$ & 0,0000 & 0,0100 & Alta \\
\hline
\end{tabular}

Fuente: Datos de las encuestas, SPSS

La ventaja relativa, mide el grado en que la adopción de Cloud Computing se percibe como mejor opción ante los programas tradicionales o actuales que poseen las PYMEs para el control de inventario.

La disponibilidad del sistema, control eficiente de la información, bajos costos de inversión y optimización del tiempo de búsqueda; corresponden a las dimensiones empleadas para el análisis de la variable. Como se observa en la tabla 2, las dimensiones de la variable tienen ponderación media-alta y alta, por lo tanto, contribuyen evidentemente a la obtención de información para la investigación. 


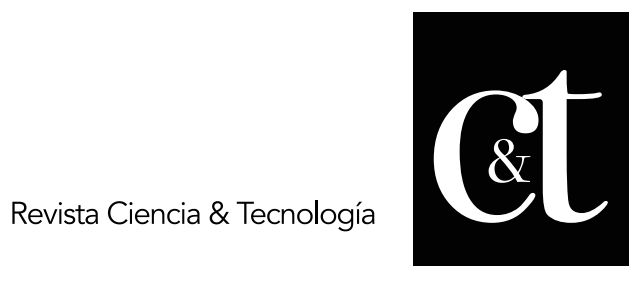

No. 32, 31 de octubre de 2021

ISSN impreso: 1390 - 6321

ISSN online: 2661 - 6734

Tabla 2: Resultado del análisis correlacional de la variable ventaja relativa y dimensiones

\begin{tabular}{|c|c|c|c|c|c|c|c|c|c|}
\hline DIMENSIÓN & RESULTADOS & & 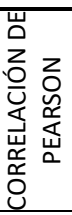 & 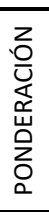 & DIMENSIÓN & RESULTADOS & & 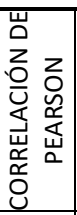 & 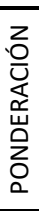 \\
\hline \multirow{5}{*}{$\begin{array}{l}\text { Disponibilidad } \\
\text { del sistema }\end{array}$} & Totalmente en desacuerdo & $7,51 \%$ & \multirow{5}{*}{0,84} & \multirow{5}{*}{$\frac{\pi}{\frac{\pi}{4}}$} & \multirow{5}{*}{$\begin{array}{l}\text { Costos Bajos } \\
\text { de Inversión }\end{array}$} & Totalmente en desacuerdo & $8,67 \%$ & \multirow{5}{*}{0,69} & \multirow{5}{*}{$\begin{array}{l}\frac{\pi}{ \pm} \\
\frac{1}{\pi} \\
\frac{\pi}{0} \\
\frac{d}{2}\end{array}$} \\
\hline & Desacuerdo & $5,20 \%$ & & & & Desacuerdo & $\begin{array}{r}12,14 \\
\%\end{array}$ & & \\
\hline & Ni acuerdo ni desacuerdo & $\begin{array}{r}15,61 \\
\% \\
\end{array}$ & & & & Ni acuerdo ni desacuerdo & $\begin{array}{r}37,57 \\
\% \\
\end{array}$ & & \\
\hline & Parcialmente de acuerdo & $\begin{array}{r}12,14 \\
\%\end{array}$ & & & & Parcialmente de acuerdo & $\begin{array}{r}17,92 \\
\%\end{array}$ & & \\
\hline & Totalmente de acuerdo & $\begin{array}{r}59,54 \\
\% \\
\end{array}$ & & & & Totalmente de acuerdo & $\begin{array}{r}23,70 \\
\% \\
\end{array}$ & & \\
\hline \multirow{5}{*}{$\begin{array}{l}\text { Control } \\
\text { Eficiente }\end{array}$} & Totalmente en desacuerdo & $3,46 \%$ & \multirow{5}{*}{0,83} & \multirow{5}{*}{$\frac{\pi}{\frac{\pi}{4}}$} & \multirow{5}{*}{$\begin{array}{l}\text { Optimización } \\
\text { del tiempo }\end{array}$} & Totalmente en desacuerdo & $3,47 \%$ & \multirow{5}{*}{0,9} & \multirow{5}{*}{$\frac{\pi}{\frac{\pi}{4}}$} \\
\hline & Desacuerdo & $1,73 \%$ & & & & Desacuerdo & $1,74 \%$ & & \\
\hline & Ni acuerdo ni desacuerdo & $\begin{array}{r}15,61 \\
\%\end{array}$ & & & & $\mathrm{Ni}$ acuerdo ni desacuerdo & $\begin{array}{r}16,18 \\
\%\end{array}$ & & \\
\hline & Parcialmente de acuerdo & $\begin{array}{r}24,86 \\
\% \\
\end{array}$ & & & & Parcialmente de acuerdo & $\begin{array}{r}28,90 \\
\% \\
\end{array}$ & & \\
\hline & Totalmente de acuerdo & $\begin{array}{r}54,34 \\
\%\end{array}$ & & & & Totalmente de acuerdo & $\begin{array}{r}49,71 \\
\%\end{array}$ & & \\
\hline
\end{tabular}

Fuente: Datos de las encuestas, SPSS

De acuerdo a los resultados obtenidos, el $71.68 \%$ de los empresarios ferreteros, consideraron estar de acuerdo que Cloud Computing cuenta con disponibilidad en sus sistemas, permitiendo llevar un control rápido y eficiente de sus inventarios, al igual que agilitar e incrementar sus ventas. De igual manera se puede apreciar que el $79.2 \%$ consideran que su uso les permite llevar un control eficiente de los procesos y el $78.61 \%$ están de acuerdo que les permite optimizar tiempo al momento de ejecutarlos. En cuanto a los costos de inversión, el $41.62 \%$ expresa que son bajos, el $37.57 \%$ no tiene conocimiento de ello y el $20.81 \%$ los considera altos.

De la información recopilada en la tabla 3 se evidencia también que el $74 \%$ de los encuestados señalaron la ventaja relativa como una variable representativa del modelo, el $77.5 \%$ coincide en la significancia de la variable compatibilidad, es decir, para los empresarios de estas ferreterías es fundamental que al decidirse por cambiar sus sistemas actuales por un sistema Cloud, éste debe ser compatible con la infraestructura, aplicaciones y procesos existentes en la empresa; el $28.3 \%$ considera al sistema complejo para su uso y el $36.4 \%$ lo considera de fácil manejo; el $19.1 \%$ ve como una limitante la seguridad del sistema, sin embargo, el $49.7 \%$ no considera inseguro implementar esta herramienta en los procesos de su empresa; el $71.7 \%$ considera importante el conocimiento previo y las capacitaciones a la hora de decidirse por la adopción, al igual que el apoyo por parte de los altos mandos de la empresa. 
Como se observa en el gráfico 4 , el $80.90 \%$ indicó que el apoyo gubernamental motivaría a la adopción por parte de las PYMEs, las regulaciones e incentivos por parte del gobierno son variables altamente importantes; el $51.45 \%$ cree que utilizar la tecnología cloud en sus procesos de inventario les permitiría obtener ventaja con la competencia; el $78.6 \%$ respondió que tener soporte oportuno de parte de los proveedores es un factor que influye en la adopción, así como también que exista diversidad de proveedores, con ofertas y servicios para poder decidir lo que más le conviene a la empresa.

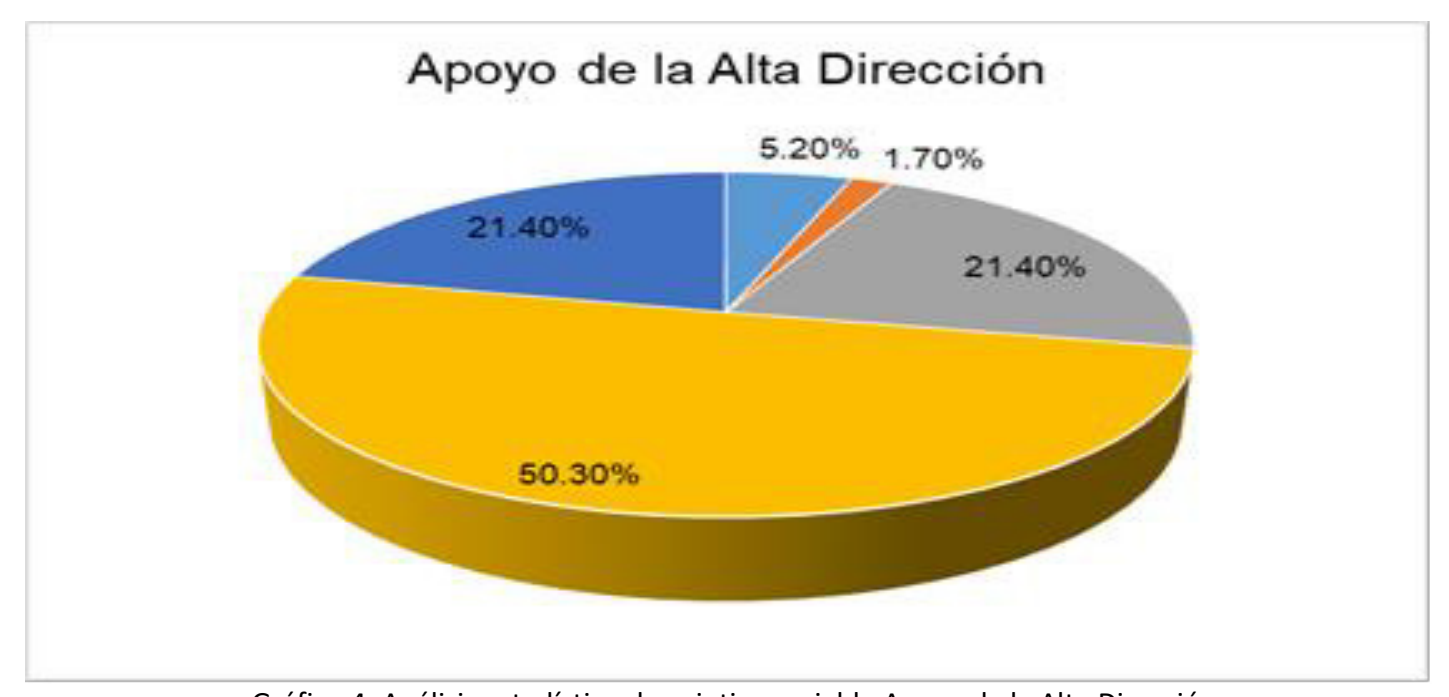

Gráfico 4: Análisis estadístico descriptivo variable Apoyo de la Alta Dirección

Fuente: Datos de las encuestas, SPSS

El $71.7 \%$ consideró que adoptar a sus sistemas de control de inventario, un sistema basado en la nube generará beneficios económicos y mejorará las habilidades internas y externas de la empresa. El apoyo de la alta gerencia, se logró constatar la importancia de la participación y anuencia de los tomadores de decisiones en las empresas, ya que son los principales motivadores sobre su equipo de trabajo, para guiarlos hacia la transición de la nueva era tecnológica. El $5.2 \%$ señalaron estar totalmente en desacuerdo; el $1.7 \%$ en desacuerdo y el $21.4 \%$, no estuvieron ni de acuerdo ni en desacuerdo con la importancia de la variable.

Cada dimensión de las variables independientes fue evaluada mediante el coeficiente de Pearson (tabla 3); todas ellas son significativas para el presente análisis. En el caso de la variable apoyo gubernamental, su dimensión "incentivo por parte del gobierno" presenta el valor más bajo, para análisis futuros podría considerarse no medirla. 


\section{Tabla 3: Resultado individual variables independientes y dimensiones}

\begin{tabular}{|c|c|c|c|}
\hline VAR INDEPEND. & DIMENSIÓN & $\begin{array}{l}\text { CORRELACIÓN } \\
\text { DE PEARSON }\end{array}$ & POND. \\
\hline \multirow{3}{*}{ COMPATIBILIDAD } & Infraestructura & 0,93 & ALTA \\
\hline & Aplicaciones & 0,88 & ALTA \\
\hline & \begin{tabular}{|l|} 
Procesos \\
\end{tabular} & 0,9 & ALTA \\
\hline \multirow{4}{*}{ COMPLEJIDAD } & Elevado esfuerzo & 0,79 & MEDIA-ALTA \\
\hline & Conectividad & 0,5 & MEDIA \\
\hline & Información compleja & 0,53 & MEDIA \\
\hline & Uso del sistema & 0,79 & MEDIA-ALTA \\
\hline \multirow{3}{*}{ SEGURIDAD } & Continuidad & 0,83 & ALTA \\
\hline & Privacidad & 0,83 & ALTA \\
\hline & Datos & 0,88 & ALTA \\
\hline \multirow{2}{*}{ PREPARACIÓN TECNOLÓGICA } & Conocimiento previo & 0,72 & MEDIA-ALTA \\
\hline & Capacitación & 0,82 & ALTA \\
\hline \multirow{3}{*}{ APOYO DE LA ALTA DIRECCIÓN } & Beneficios percibidos en las habilidades & 0,74 & MEDIA-ALTA \\
\hline & Beneficios económicos percibidos & 0,76 & MEDIA-ALTA \\
\hline & Recursos para la adopción & 0,64 & MEDIA-ALTA \\
\hline \multirow{2}{*}{ APOYO GUBERNAMENTAL } & Regulación & 0,54 & MEDIA \\
\hline & Incentivo & 0,08 & BAJA \\
\hline PRESIÓN DE LA COMPETENCIA & Competencia & NO APLICA & NO APLICA \\
\hline \multirow{2}{*}{ PROVEEDOR DE SERVICIOS } & Diversidad de proveedores & 0,78 & MEDIA-ALTA \\
\hline & Soporte Oportuno & 0,87 & ALTA \\
\hline
\end{tabular}

Fuente: Datos de las encuestas, SPSS

Con el propósito de fundamentar la adopción del Cloud Computing, se realizó un análisis económico, a través de los indicadores económicos; Costo Total de la Propiedad (TCO) y Retorno de la Inversión (ROI), observados en las tablas 4 y 5.

Tabla 4: Costo Total de Propiedad

\begin{tabular}{lrr}
\hline \multicolumn{3}{c}{ TCO Costo Total de Propiedad } \\
\hline & On-Premises & Cloud Computing \\
Hardware & 1745,5 & \\
Servicio contratado & 3860,67 & 975,33 \\
Licenciamiento & 1085,28 & 611,63 \\
Soporte & & \\
Energía & 118,92 & \\
Mantenimiento & 200 & $\mathbf{1 5 8 6 , 9 6}$ \\
\hline Total & $\mathbf{7 0 1 0 , 3 7}$ & $\mathbf{5 4 2 3 , 4 1}$ \\
\hline Total Ahorrado & & $\mathbf{7 7 \%}$
\end{tabular}

Fuente: Proformas de proveedores del servicio 


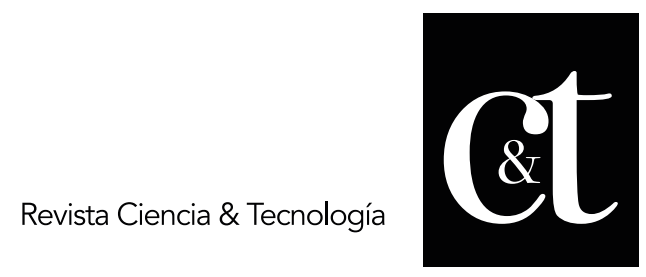

No. 32, 31 de octubre de 2021

ISSN impreso: 1390 - 6321

ISSN online: 2661 - 6734

Tabla 5: Retorno de la Inversión

\begin{tabular}{|c|c|c|c|}
\hline & & Año 1 & $\begin{array}{r}\text { Total } 5 \\
\text { años }\end{array}$ \\
\hline Ganancia & $\$$ & $5.423,41$ & $\begin{array}{r}\$ \\
7.345,91\end{array}$ \\
\hline Costo & $\$$ & $1.586,96$ & $4.033,47$ \\
\hline $\begin{array}{l}\text { ROI } \\
\text { (Dólares) }\end{array}$ & $\$$ & $3.836,45$ & $\begin{array}{r}\$ \\
3.312,45\end{array}$ \\
\hline ROI (\%) & & $242 \%$ & $82 \%$ \\
\hline
\end{tabular}

Fuente: elaboración propia

Evaluando los costos de implementación de Cloud Computing en comparación con un sistema On-Premises, las PYMEs podrán ahorrar un estimado del $77 \%$ en el primer año; las empresas se ahorran gastos en hardware, licenciamiento, energía eléctrica y mantenimiento de equipos; incluso, los costos por instalación del servicio y soporte técnico son más bajos en comparación de las soluciones locales. En consecuencia, de las ganancias que obtendrían al implementar un sistema basado en la nube y el ahorro generado; el retorno de la inversión en el primer año es del $242 \%$, casi 3 veces más alto que la inversión inicial; se debe, en gran parte, en que las empresas se evitarían adquirir equipos informáticos, licenciamiento u otros equipos necesarios para la instalación.

En una proyección a cinco años el ROI es del $82 \%$, evidentemente mucho más bajo que en el año 1 , porque ya no se requiere de la inversión inicial, pese a ello, el valor sigue siendo representativo.

\section{Conclusiones}

El sector ferretero de la ciudad de Machala está compuesto en su mayor parte por micro, pequeñas y medianas empresas, que, por su tamaño, nivel organizacional o falta de recursos desconocen de los beneficios que genera la implementación de un sistema de control de inventario basado en Cloud Computing. Sin embargo, se demostró mediante la aplicación de las encuestas, que las PYMES poseen la intención de implementarlo y requieren de mayor información, acompañamiento e incentivos que las motiven a decidirse. Las variables independientes analizadas dentro del contexto tecnológico y organizacional seleccionadas en el modelo, demostraron ser altamente significativas con un nivel de significancia entre 0.001 y 0.010 ; los valores de Chi2 son positivos, indicando la relación de las variables independientes con la variable dependiente. 


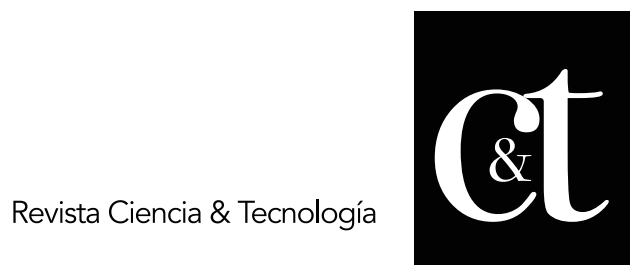

No. 32, 31 de octubre de 2021

ISSN impreso: 1390 - 6321

ISSN online: 2661 - 6734

Por lo tanto, generar investigaciones que contribuyan a estas empresas, a facilitar el proceso de adopción es fundamental. A través de los modelos de adopción de tecnología, se obtiene una herramienta para la identificación de las variables; el modelo TOE es clave para la obtención de resultados que permitieron el desarrollo del estudio, eso no quiere decir que sea el único modelo o el más adecuado para otros trabajos a realizarse en el futuro; las variables consideradas fueron de acuerdo a la percepción que se evidenció de la situación actual de las PYMEs del sector.

Por otro lado, el apoyo de la alta gerencia es fundamental para la adopción; en las manos de los gerentes o propietarios de las PYMEs, está la decisión de remplazar los sistemas actuales e implementar un sistema basado en la nube, considerando la realidad que atraviesa el mundo, donde hoy en día prevalecen las empresas que se encuentran más cerca de las innovaciones tecnológicas.

Contribuyendo con los empresarios en la toma de decisiones, se ofreció un análisis de los costos y ganancias de sistemas basados en la nube, respecto a los sistemas On-Premises; las empresas pueden aplicar estos cálculos con los costos y gastos de sus sistemas actuales en relación con las ofertas que existan en el mercado de Cloud Computing y generar su propio análisis.

\section{Referencias bibliográficas}

Aljabre, A. (2012). Cloud computing for increased business value. Journal of Business and Social Science, 3(1), 234-240.

Alomoto, N., Acuña, C., Salvador, M., Ortíz, J., \& Ruiz, A. J. (2014). LA GESTIÓN DE LA CADENA DE SUMINISTRO EN ECUADOR: CASO DE LAS PyMEs. In Revista Arbitrada Formación Gerencial (Vol. 13, Issue 2). Universidad del Zulia.

Armbrust, M., Fox, A., Griffith, R., Joseph, A., Katz, R., Konwinski, A., Lee, G., Patterson, D., Rabkin, A., Stoica, I., \& Zaharia, M. (2010). A view of cloud computing. International Journal of Networked and Distributed Computing, 53(4). https://doi.org/10.2991/ijndc.2013.1.1.2

Celleri, J., Rivas, W., Andrade, J., \& Rodriguez, S. (2018). Análisis del uso del Cloud Computing en empresas de Ecuador. Alternativas, 19(2), 69-73. https://doi.org/10.23878/alternativas.v19i2.251

Dean, D., \& Saleh, T. (2010). Captar el verdadero valor del 'cloud computing.' Harward Deusto Business Review, 188, 34-48.

Dubey, A., \& Wagle, D. (2007). Delivering software as a service. The McKinsey Quarterly, 6.

Ercolani, G. (2012). Análisis del potencial del Cloud Computing para las PYMEs. Dialnet.

Ercolani, G. (2017). Análisis del potencial del cloud computing para las PYMES : un modelo integrado para evaluar software as a service (SaaS) en la nube pública.

Indahningrum, R. putri. (2020). MODELO DE ADOPCION PARA EL USO DEL CLOUD COMPUTING EN LAS PYMES DEL SECTOR EXPORTADOR DE CAMARON DEL CANTON DURAN. 2507(1), 1-9.

Jimenez, D. (2013). La "computación en la nube" o "cloud computing" examinada desde el ordenamiento jurídico español. Revista de Derecho de La Pontificia Universidad Católica de Valparaíso XL. 


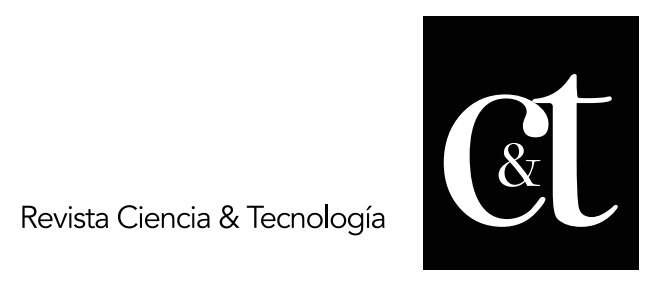

No. 32, 31 de octubre de 2021

ISSN impreso: 1390 - 6321

ISSN online: 2661 - 6734

Mariscal, J., \& Gil, R. (2013). El computo en la nube en México: Alcances y Desafíos para los Sectores Público y Privado. Cide, 280, 24.

Mazon, B., Jaramillo, M., Romero, O., Aguirre, M., Ruiz, J., \& Eras, J. (2018). Modelo de Preparación de las TIC y Adopción del E-commerce en el Sector $\begin{array}{llll}\text { Comercio (PTACE). Revista } & \text { Espacios, }\end{array}$ http://www.revistaespacios.com/a18v39n24/18392415.html

Mell, P., \& Grance, T. (2011). The NIST Definition of Cloud Computing (Vol. 514). https://doi.org/10.1007/978-981-13-1056-0_66

Mohlameane, M., \& Ruxwana, N. (2014). The Awareness of Cloud Computing: A Case Study of South African SMEs. International Journal of Trade, Economics and Finance, 5(1). https://doi.org/10.7763/ijtef.2014.v5.332

Otake, L. A. (2019). " MODELO PARA LA ADOPCIÓN DE TECNOLOGÍAS DE LA INFORMACIÓN EN LAS PEQUEÑAS Y MEDIANAS EMPRESAS DE LAMBAYEQUE - PERÚ ." Universidad Científica del Sur.

Pérez, D., Solana, P., \& Trigueros, S. (2018). Economía del dato y transformación digital en pymes industriales: Retos y oportunidades. Economía Industrial, 409, 37-45. https://repositorio.unican.es/xmlui/bitstream/handle/10902/15123/Economí aDatoTransformación. pdf?sequence $=1$

Ruivo, P., Rodrigues, J., \& Oliveira, T. (2015). The ERP Surge of Hybrid Models An Exploratory Research into Five and Ten Years Forecast. Procedia Computer Scienceel, 64, 594-600. https://doi.org/10.1016/j.procs.2015.08.572

Salleh, S. M., Teoh, S. Y., \& Chan, C. (2012). Cloud enterprise systems: A review of literature and its adoption. Proceedings - Pacific Asia Conference on Information Systems, PACIS 2012.

Vidhyalakshmi, P., \& Kumar, V. (2016). Determinants of cloud computing adoption by SMEs. International Journal of Business Information Systems, 22(3), 375395. https://doi.org/10.1504/IJBIS.2016.076878 